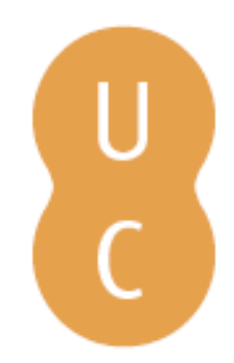

\title{
pommalina
}

\section{I poeti tragici maestri di virtù nelle opere di Plutarco}

\author{
Autor(es): $\quad$ Carrara, Paolo
}

Publicado por: Imprensa da Universidade de Coimbra; Katholieke Universiteit Leuven

URL

persistente: URI:http://hdl.handle.net/10316.2/32095

DOI: $\quad$ DOI:http://dx.doi.org/10.14195/978-989-26-0462-6_5

Accessed : $\quad$ 26-Apr-2023 12:03:29

A navegação consulta e descarregamento dos títulos inseridos nas Bibliotecas Digitais UC Digitalis, UC Pombalina e UC Impactum, pressupõem a aceitação plena e sem reservas dos Termos e Condições de Uso destas Bibliotecas Digitais, disponíveis em https://digitalis.uc.pt/pt-pt/termos.

Conforme exposto nos referidos Termos e Condições de Uso, o descarregamento de títulos de acesso restrito requer uma licença válida de autorização devendo o utilizador aceder ao(s) documento(s) a partir de um endereço de IP da instituição detentora da supramencionada licença.

Ao utilizador é apenas permitido o descarregamento para uso pessoal, pelo que o emprego do(s) título(s) descarregado(s) para outro fim, designadamente comercial, carece de autorização do respetivo autor ou editor da obra.

Na medida em que todas as obras da UC Digitalis se encontram protegidas pelo Código do Direito de Autor e Direitos Conexos e demais legislação aplicável, toda a cópia, parcial ou total, deste documento, nos casos em que é legalmente admitida, deverá conter ou fazer-se acompanhar por este aviso.

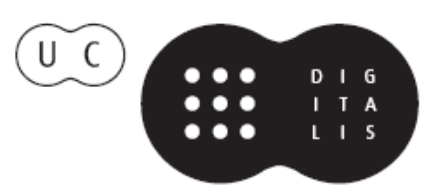




\title{
Puilosophy in Society \\ Virtues and Values in Plutarch
}

\author{
José Ribeiro Ferreira \\ LUC VAN DER STOCKT \\ Maria do Céu Fialho
}

Editors

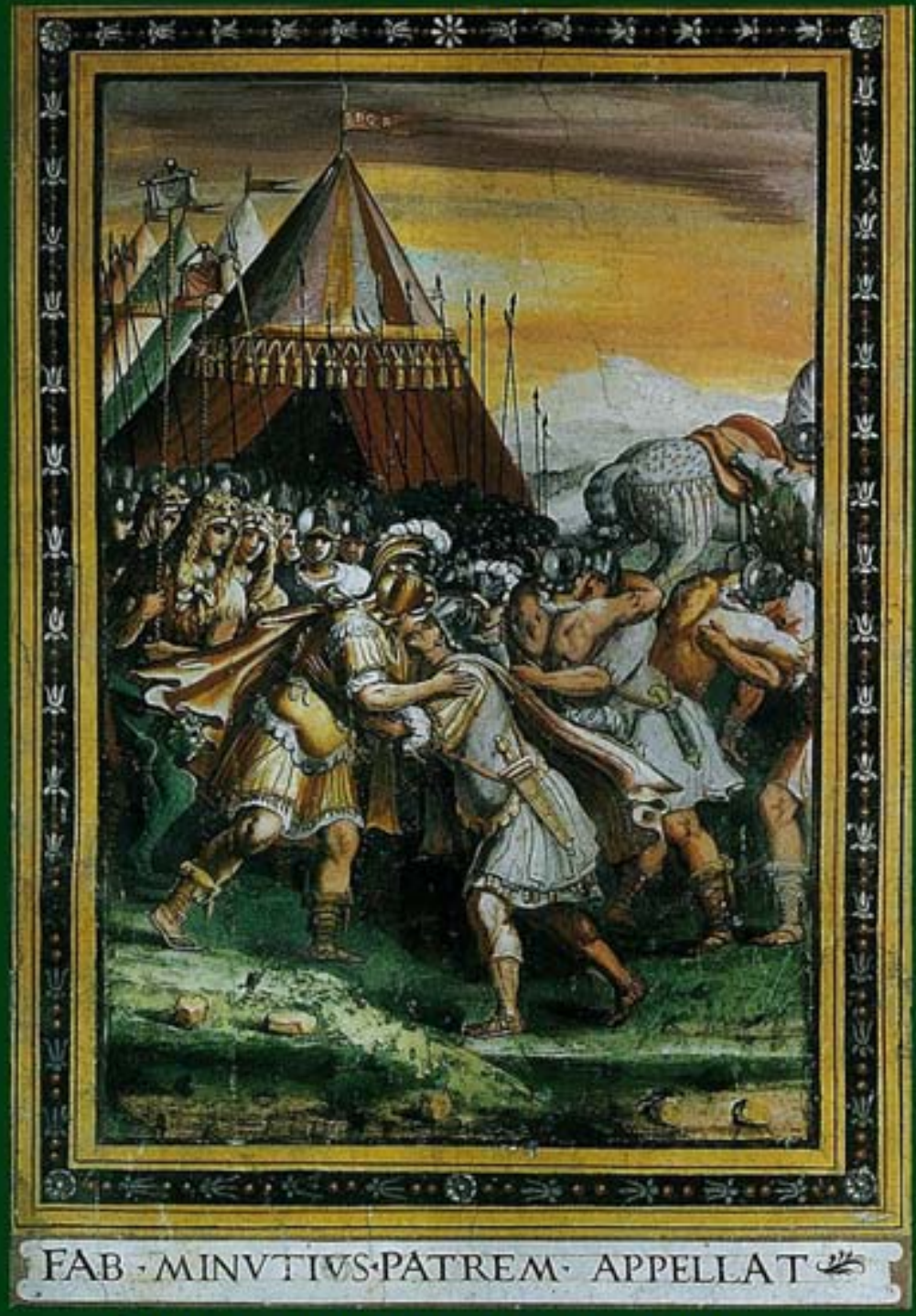

KATHOLIEKE UNIVERSITEIT LEUVEN IMPRENSA DA UNIVERSIDADE DE COIMBRA Leuven-Coimbra, 2008 
(Página deixada propositadamente em branco) 
José Ribeiro Ferreira, Luc van der Stockt \& Maria do Céu Fialho

EDITORS

\section{PhILOSOPHY IN Society}

\section{Virtues and Values in Plutarch}

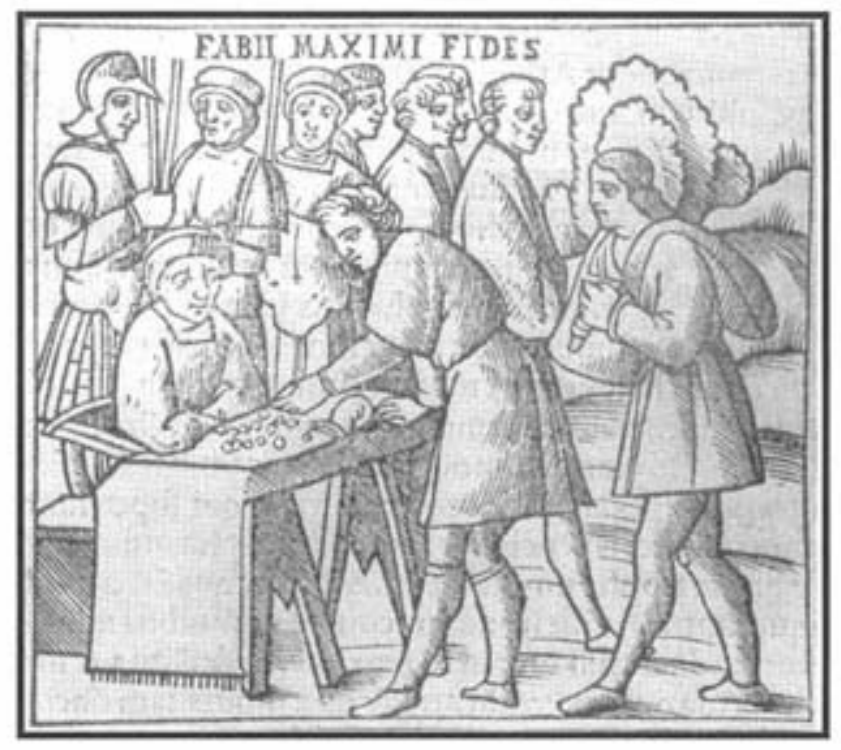

Fabius Maximus' Loyalty

Vitae Plutarchi Cheronei novissime post Jodocum Badium Ascensium longe diligentius repositae maioreque diligentia castigatae, cum copiosiore verioreque indice, nec non cum Aemilii Probi vitis, una cum figuris, suis locis apte dispositis, Venetiis 1516 , fol . 65v

\section{LEUVEN - CoIMBRA \\ 2008}

KATHOLIEKE UNIVERSITEIT LEUVEN

IMPRENSA DA UNIVERSIDADE DE COIMBRA 


\title{
First published 2008
}

\section{(C) Universiteit KATHOlieke Leuven \\ (c) Universidade Coimbra}

\author{
Published by \\ IMPRENSA DA UNIVERSIDADE COIMBRA \\ Imprensa da Universidade de Coimbra \\ Rua da Ilha, $\mathrm{n}^{\circ} 1$ \\ 3000-033 Coimbra (Portugal) \\ Email: imprensauc@ci.uc.pt \\ URL: http://www.uc.pt/imprensa_uc
}

ISBN: 972-989-8074-73-7

Legal Deposit: MA-140-2009

\author{
Printed in Spain by \\ IMAGRAF IMPRESORES, S.A. \\ c/ Nabucco 14 \\ 29006 Málaga \\ Tfno. 952328597
}

\section{Frontispiece:}

Fabius Maximus and Minucius (Francesco da Siena, Grottaferrata, Palazzo Abbaziale). We are grateful to the Archimandrita of the "Monastero Esarchico di Santa Maria di Grottaferrata", P. Emiliano Fabbricatore, for the authorization to reproduce this picture. 


\section{I poeti tragici maestri di virtù nelle opere di Plutarco \\ PaOlo Carrara \\ UNIVERSITÀ DI SIENA}

Nella vasta opera di Plutarco numerosissime sono le citazioni di poeti antichi, in particolare di Omero e dei tragici ${ }^{1}$. Non di rado queste citazioni sono puramente decorative, artifici eleganti per variare e movimentare stilisticamente la dizione letteraria. In altri casi sono citazioni di luoghi celeberrimi, passati quasi a proverbio, e se anche molto spesso di essi si è persa la esatta nozione della paternità, risuonano tuttavia nell'orecchio di tutti.

C'è però un altro, e forse più importante significato che le citazioni poetiche assumono negli scritti plutarchei. La produzione poetica greca infatti è, per il tradizionalista e "romantico" Plutarco, anche l'antico retaggio della cultura nazionale, di quella cultura che stava proprio in quel tempo combattendo una importante battaglia, che avrebbe deciso della sua sopravvivenza e, inscindibilmente, della sua supremazia, oppure del suo definitivo declino di fronte alla cultura romana. Un carattere fortemente qualificante di questo patrimonio culturale è costituito, per Plutarco, certamente da una caratura di alto valore estetico. Egli tuttavia amò sempre presentarsi, e certamente così si percepiva, prima ancora che come fine letterato, soprattutto come un "filosofo", come un uomo colto che identificava nella filosofia, secondo il senso che essa aveva assunto ormai da qualche secolo, il vertice della formazione umana e sociale della persona. Pertanto il vero valore della tradizione ellenica consisteva, per lui, non tanto nel raggiungimento di vette di eccel-

1 Per le citazioni in Plutarco si veda W. C. HeLmBold - E. N. O'NeIL, Plutarch 's quotations, American Philological Association, New York, 1959. Per una valutazione complessiva delle citazioni dei poeti tragici in Plutarco si veda L. DI Gregorıo, "Plutarco e la tragedia", Prometheus, 2 (1976) 151-174; ID., "Lettura diretta e utilizzazione di fonti intermedie nelle citazioni plutarchee dei tre grandi tragici, I", Aevum, 53 (1979) 11-50; II, Aevum, 54 (1980) 46-79.

José Ribeiro Ferreira, Luc Van der Stockt \& Maria do Céu Flalho (Edd.), Philosophy in Society - Virtues and Values in Plutarch, Leuven-Coimbra, 2008, pp. 65-74. 
lenza sotto il profilo estetico, quanto soprattutto nel primato in quel campo che appunto si definiva filosofia: un insieme di ambiti che, senza ovviamente trascurare la pura ricerca teoretica, acquista il suo vero significato e valore nel realizzarsi in campo scientifico e dottrinale culminando nella esperienza morale e religiosa ${ }^{2}$.

Plutarco dunque, come filosofo, si serve molto spesso, in tutti i suoi scritti ed in particolare in quelli di carattere più spiccatamente morale, della citazione poetica come veicolo di pensieri etico-filosofici, sia per dare maggior rilievo e vigore ad una affermazione o ad un pensiero attraverso la forma poetica, sia perché egli attinge dal patrimonio culturale della nazione, che, specialmente nel passato più lontano, si era espresso in termini poetici ${ }^{3}$. Nel fare ciò, egli si conforma all'uso diffuso nella letteratura filosofico-morale dei suoi tempi: Cicerone e Seneca, ad esempio, prima di lui, procedettero in modo analogo. Del resto l'impiego di testi poetici come parametri di conforto e sostegno ad affermazioni filosofiche è antico: lo troviamo già in Platone. Gli Stoici, poi è noto, usarono ampiamente estratti dai poeti per suffragare le loro teorie, sviluppando anche una metodologia dell'antologizzazione, sulla quale torneremo ${ }^{4}$.

Plutarco si rivolge, nelle sue citazioni, a tutto il patrimonio della letteratura greca, soprattutto arcaica e classica, ma anche, sebbene in misura minore, ellenistica. Non sfuggirà comunque al lettore che la gran massa dei passi sono presi da Omero e dai tragici: Euripide in modo particolare. Il catalogo di Helmbold-O'Neil, sebbene bisognoso di qualche integrazione ${ }^{5}$, ci permette tuttavia di farci una buona idea di questa situazione.

Cfr. L. Di Gregorio, "Plutarco e la tragedia", Prometheus, 2 (1976) p. 157.

Plutarco ha naturalmente ben presente la distinzione aristotelica (cfr. Poet. 1, 1447 b, 17-20) fra poesia e opere semplicemente composte in versi e se ne serve. Ad esempio egli cita spesso Empedocle trattandolo come filosofo e contrapponendolo, in positivo, ai poeti.

Antologie e gnomologi si dovettero formare all'epoca dei grandi tragici o subito dopo; un riflesso del loro impiego può intravedersi giả nel modo di procedere di Platone, il quale si serve, nelle proprie argomentazioni, di sentenze, tratte dai tragici, non necessariamente contestualizzate. Sulla formazione e sulla storia di antologie e gnomologi si vedano sempre i classici A. ELTER, De gnomologionum Graecorum historia atque origine, Progr. Ac. Bonn. 1893-1894; K. HoRnA, in R.E. Supplb. 6, 1935, coll. 74-87, e le utili riflessioni e messe a punto sui vari generi di J. BARNS, "A new Gnomologium with some Remarks on gnomic Anthologies", $C Q, 44$ (1950) 126-137; 45 (n. s. 1) (1951) 1-19. Si vedano anche i più recenti: R. M. PICcione, "Sammeln, Neuordnen, Neues Shaffen", in Selecta colligere, hrsg. von R. M. PICcione und M. Perkams, 1, Alessandria, 2003, pp. vIl-XV; EAD., Le raccolte di Stobeo e Orione. Fonti, modelli, architetture, in Aspetti di letteratura gnomologica nel mondo antico, a cura di M. S. FunGHI, I, Firenze, 2003, pp. 241-261; Ead., "Forme di trasmissione della letteratura sentenziosa", in Aspetti di letteratura gnomologica nel mondo antico, a cura di M.S. Funghi, II, Firenze 2004, pp. 403-441.

Cfr. V. CrrTI, "Eschilo in Plutarco", in La biblioteca di Plutarco. Atti del IX congresso plutarcheo (Pavia, 13-15 giugno 2002), a cura di I. Gallo, Napoli, 2004, pp. 135-145. 
Fin qui dunque nulla di strano nella prassi del dotto di Cheronea.

Se tuttavia riflettiamo sulle posizioni filosofiche plutarchee, un problema di ordine teorico sembra nascere immediatamente, ed è stato notato spesso dagli studiosi. Plutarco infatti, pur accogliendo, nelle proprie posizioni filosofiche, secondo la prassi diffusa nella cultura del suo tempo, dovuta anche a l'influenza di altre tradizioni speculative, soprattutto quelle di matrice aristotelica, si professò sempre un platonico ${ }^{6}$ e alla filosofia di Platone, da lui accuratamente studiata fino dalla giovinezza, dedicò anche alcune opere specifiche, quali le Quaestiones Platonicae e il De procreatione animae in Timaeo ${ }^{7}$. Ora è noto come Platone, uomo certamente sensibilissimo al fascino della poesia, avesse tuttavia decretato, nella Repubblica ${ }^{8}$, una sorta di ostracismo nei confronti della poesia, soprattutto epica e tragica, quella cioè di gran lunga più praticata e influente al suo tempo. La condanna platonica si fonda soprattutto su due constatazioni che renderebbero la poesia dannosa e "politicamente scorretta". La prima scaturisce da un motivo di ordine teoretico-dot-

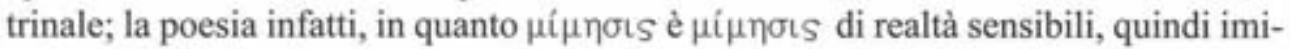
tazione dell'imitazione, e pertanto completamente destituita di ogni contenuto speculativo e filosofico.

Vi è però, molto più diffusamente esaminato, un motivo di ordine morale, cui Platone attribuisce un peso grandissimo. I poeti, proprio per questa lontananza dal reale cioè dall'idea, intessono di menzogne i loro $\mu$ ṽ$o t$ sugli dei e sugli eroi; essi non possiedono la verità razionale su Dio, sull'uomo e sul mondo; la loro teologia, cosmologia e antropologia sono difettose e bugiarde, frutto di inganni molteplici, per non dire, di conseguenza, dei loro insegnamenti pratici, spesso ispirati ad un sistema di valori fallace. I poeti tragici in particolare, a cominciare da Euripide, esaltano la tirannide e dai tiranni sono remunerati: per questo meritano di essere espulsi dalla repubblica ${ }^{9}$.

Sulle idee filosofiche plutarchee e la loro formazione cfr., oltre al classico K. ZiEGLer, Plutarco (Stuttgart, 1949), trad. it. Brescia, 1965, pp. 361-369, F. FerRARI, Dio, idee e materia. La struttura del cosmo in Plutarco, Napoli. 1995; ID., "Platone in Plutarco", in La biblioteca di Plutarco. Atti del IX convegno plutarcheo (Pavia, 13-15 giugno 2002), a cura di I. Gallo, Napoli, 2004, pp. 225-35; F. BECCHI, "Plutarco tra platonismo e aristotelismo: la filosofia come paideia dell'anima, in Plutarco, Platón y Aristóteles", Actas del V Congreso Internacional de la L.P.S. (Madrid-Cuenca, 4-7 de Mayo de 1999), Madrid, 1999, pp. 25-43.

7 Anche la polemica contro altre scuole filosofiche, che sostanzia le opere antiepicuree e antistoiche, è condotta costantemente da posizioni platonico-aristoteliche.

$8 \mathrm{Si}$ veda in particolare l'ampia trattazione del filosofo in Resp. II, 17-21, 376e-383c. Cfr. S. GAStALDI, "Paideia/mitologia", in Platone, La repubblica, a cura di M. VegetT, II (libb. I-II), Napoli., 1998, pp. 333-392.

Cfr. Resp. VIII, 18, 568 b-d. Proprio in quanto presunti propugnatori della tirannide "aùTois $\epsilon$ is

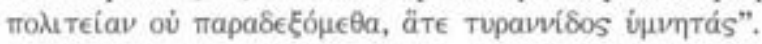


La posizione platonica, sappiamo, non trovò seguito incondizionato nell'antichità. Diffidenza nei confronti della poesia troviamo negli epicurei, ma originata da motivazioni in gran parte molto diverse, mentre Aristotele e gli Stoici non pervennero affatto alla condanna radicale della poesia, ma anzi la studiarono e se ne servirono.

Come si muove Plutarco in questo quadro? Abbiamo visto che tutta l'opera plutarchea letteralmente gronda di poesia, essa è tutta intessuta da un capo all'altro di citazioni, reminiscenze, echi della poesia soprattutto omerica e tragica. Quale risposta, si è invocato spesso il concetto di "eclettismo": Plutarco prenderebbe da Platone quello che gli piace e da altri filosofi altri elementi. Questo giudizio, espresso in questi termini, sarebbe troppo semplicistico. Si è anche messo in campo una sorta di dicotomia nel pensiero di Plutarco: negli scritti e nei passaggi teorici egli si atterrebbe alla dottrina platonica e quindi alla condanna della poesia, che invece utilizzerebbe, sul modello Stoico, in sede pratica ${ }^{10}$.

In particolare, proprio la tragedia sarebbe nel mirino della condanna plutarchea quando affronta il tema su basi teoriche. Quindi egli condannerebbe con Platone e a partire da Platone la tragedia, servendosene poi all'occasione in sede pratica.

Ovviamente la situazione è più complessa e soprattutto molto più articolata. Il testo, infatti, normalmente citato a sostegno di un Plutarco che, sulla scia di Platone, condannerebbe la tragedia è costituito da alcuni capitoli centrali di uno scritto retorico giovanile ${ }^{11}$, il De gloria Atheniensium. Si tratta dei cap. 5-7 ${ }^{12}$.

In questi capitoli, Plutarco svolge la tesi, di sapore scolastico - come scolastico è tutto lo scritto, una declamazione retorica - secondo la quale gli Ateniesi non si sarebbero distinti nella poesia epica e lirica, e se attenzione dedicarono alla poesia drammatica, ciò fu piuttosto sintomo e frutto di una certa degenerazione. Degenerazione corretta tuttavia dal fatto che essi avrebbero sempre anteposto alla ricerca e all'apprezzamento della gloria letteraria, quelli per la gloria militare e politica. Come si vede, leggendo questi capitoli, il riferimento a Platone, indicato dal Di Gregorio, non c'è in questo luogo. Di Platone, è vero, si parla subito prima, nel capitolo IV, ricordando, in riassunto, la sua celebre condanna nei confronti della poesia.

Che la poesia sia una produzione di miti, lo ha detto anche Platone. Il mito vuole essere un discorso mendace ( corso vero. Pertanto esso è molto distante dai fatti, dal momento che il dis-

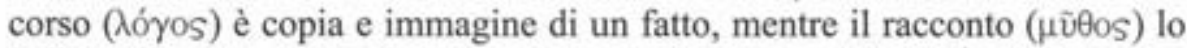
è di un discorso. Tanto dunque coloro che inventano i fatti (oi $\pi \lambda a ́ t T O \nu T \in S$

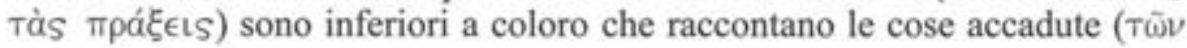

Cfr. K. Ziegler, Plutarco, cit., p. 115.

Cfr. L. Di Gregorio, in Aevum, 53 (1979) p. 11. 


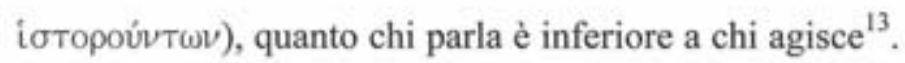

Seguono i capp. 5-7 di cui si è detto. Come si vede il richiamo a Platone è molto rapido e, sebbene preciso, assai poco sviluppato da un punto di vista teoretico: quasi una parentesi. E questo ovviamente non perché a Plutarco mancasse la cognizione approfondita della dottrina di Platone, ma perché qui egli è interessato a svolgere un altro tipo di argomentazione $\mathrm{e}$ in un campo e registro diverso.

Nei capitoli 5-7, infatti, assistiamo da parte di Plutarco, ad una totale svalutazione della commedia, composizione indegna di un gentiluomo ${ }^{14}$. Egli passa quindi ad occuparsi della tragedia, chiedendosi le ragioni del suo successo:

Fiori la tragedia e divenne famosa, facendosi motivo di ascolto e di spettacolo per gli uomini di quel tempo e offrendo, tramite i miti e le passioni, un inganno ${ }^{15}$, come dice Gorgia, per il quale l'ingannatore è più giusto del non

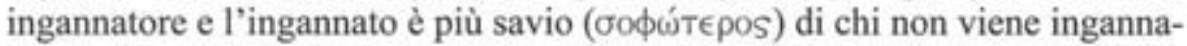
to. L'ingannatore infatti è più giusto, in quanti realizza ciò che aveva promesso. E l'ingannato più savio ${ }^{16}$.

E' Plutarco stesso a dirci che questa ingegnosa definizione è di Gorgia ( $\dot{\omega}$ s Гopyias $\phi \eta \sigma i v)^{17}$, e questo fatto potrebbe essere la chiave per intendere meglio il vero senso e la matrice di siffatta specifica condanna di Plutarco nei confronti della tragedia, quale leggiamo nei capp. 5-9 del De gloria Atheniensium. Una condanna che punta i suoi strali più feroci, non già sull'opera dei tragediografi, ma sulla volgarità della pompa degli allestimenti tragici, sulle bizzarrie degli attori e sul costo esagerato degli spettacoli ${ }^{18}$. Come si vede, tutto ciò ha ben poco di platonico e proviene con ogni probabilità da una topica di ascendenza retorico-sofistica - la citazione di Gorgia appunto mi pare che illumini l'ambito di provenienza - che doveva

13

14

15

16

17

18

Plut., De glor: Athen. 4, 348 A-B.

Troviamo qui (De glor: Athen. 5, 348 B) una curiosa notizia, nota dal solo Plutarco, secondo la quale agli Areopagiti sarebbe stato vietato da una legge di dedicarsi alla composizione di commedie.

La paternità gorgiana della definizione sembra garantita, anche nel suo aspetto formale, dal gioco

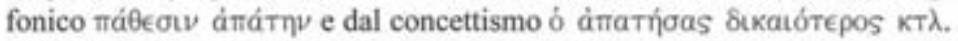

Plut. De glor: Athen. 5, $348 \mathrm{C}$.

Cfr. 82 B 23 D-K.

Cfr. De glor: Athen. 5-6, 348 C - 349 B; l'aneddoto dello Spartano indignato di fronte allo sfarzo degli spettacoli ateniesi (cfr. De glor. Athen. 6, 348 F) si legge anche in Quaest. Conv, 7, $710 \mathrm{~F}$ 711 A. Le critiche che Plutarco muove sono in gran parte da intendersi dirette contro la tragedia postclassica: l'accenno alla preponderanza dell'apparato visivo e alle stravaganze degli attori si spiegano infatti meglio se riferite alla tragedia dei secoli successivi al quinto. Si veda anche Plutarco, La gloria di Atene, a cura di I. GALto - M. Moccl, Napoli, 1992, p. 100, nota 70. 
trovare ampio spazio nell'armamentario professionale delle scuole di retorica e come tale essere stata impiegata dal giovane Plutarco.

In sostanza, la condanna platonica della tragedia non sembra aver radicalmente coinvolto Plutarco ed averlo profondamente condizionato. Egli conosce bene, come si è visto, la teoria dell'imitazione, ma di essa non sembra giovarsi particolarmente in questo luogo per sostenere la propria argomentazione, che, come si è rilevato, sembra avere altre sorgenti. Dopo Platone, infatti, Aristotele aveva rivalutato la poesia, e in particolare la tragedia, non solo sul piano estetico, ma, quello che a noi qui più interessa, anche sul piano etico e psicologico: la dottrina della catarsi ${ }^{19} \mathrm{è} \mathrm{un}$ esempio lampante di ciò. Plutarco dunque, qui e in molti altri luoghi della sua opera, non sembra particolarmente sensibile al problema teoretico dello status della poesia e alla soluzione platonica al problema.

Molto più forte e produttivo è in lui il senso della tradizione greca, tradizione della quale la grande poesia fa ormai inscindibilmente parte.

Il problema sembra, per Plutarco, essere piuttosto il seguente: che rapporto intercorre fra poesia e filosofia? E secondariamente: cosa si può ricavare dalla poesia tragica in ordine ad una formazione filosofica della persona?

Egli, in altre parole, ripropone il quesito platonico circa il contenuto morale della poesia e circa l'eventuale ricaduta della frequentazione della poesia sulla vita di tutti i giorni, senza che l'accento batta troppo sulla domanda, teoretica, se e quanto di $\dot{a} \lambda \hat{\theta} \theta \in \iota a$ vi sia nella poesia stessa.

A questo proposito possiamo rivolgere l'attenzione alla lettura di un celeberrimo scritto plutarcheo, celebre quanto forse spesso malamente inteso: l'operetta nota comunemente sotto il titolo De audiendis poetis. Intanto, già il titolo vulgato, in latino, indirizza erroneamente verso il contenuto del trattatello. Nei manoscritti greci

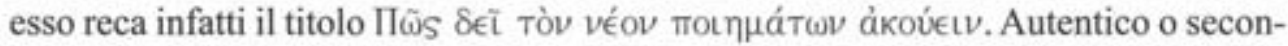
dario che sia, questo titolo già ci orienta per collocare con maggior precisione lo scritto. Non si tratta dunque né di un trattato di poetica, alla maniera della Poetica aristotelica per intenderci, né di estetica, né infine di critica letteraria. Evidentemente i convincimenti estetici e critico-letterari dell'autore sono una premessa ovvia e imprescindibile per collocare correttamente molte delle sue affermazioni e delle sue scelte, e ad esse Plutarco accenna soprattutto nella sezione introduttiva dell'opera. Il trattato, però, ha come fine esplicito quello di chiarire il ruolo e il peso, e di conseguenza le modalità, che la lettura dei poeti - ed è bene ancora ricordarlo che si tratta soprattutto di Omero e dei tragici - debba avere nell'educa-

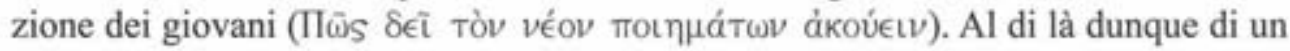


dibattito sulla natura della poesia, cui, come si è detto, Plutarco accenna nelle premesse, il nucleo della questione è se, ed eventualmente come, il giovane possa e debba leggere i poeti.

Plutarco, ovviamente, ritiene come punto fermo che la formazione del giovane debba risolversi, in prospettiva, in un percorso che condurrà alla filosofia; il suo problema, pertanto, è di verificare secondo quale opportunità e con quali precise modalità la lettura dei poeti possa inserirsi in questo percorso.

Il presupposto e allo stesso tempo la sintesi della soluzione che Plutarco prospetta è senza dubbio grandemente influenzata dall'analisi platonica, con i due esiti che abbiamo menzionato:

la poesia è imitazione dell'imitazione;

la poesia contiene menzogne e dottrine moralmente nocive.

Il primo punto rimane sostanzialmente sullo sfondo e Plutarco, pur conoscendolo bene $\mathrm{e}$ in qualche modo presupponendolo, non lo sviluppa qui approfonditamente. Che la poesia fosse meno "vera" della filosofia è un dato ormai acquisito per la tradizione che a Platone fa capo. Il secondo punto, che quanto ad analisi coincide perfettamente con la posizione platonica, propone tuttavia una soluzione diversa: la poesia contiene del vero e del falso, quindi può essere utile strumento di educazione se la sua lettura sarà convenientemente pilotata. La poesia infatti possiede un fascino particolare, suo proprio, che utilmente può essere sfruttato a fini pedagogici. L'esempio dell'impiego della testa del polpo come cibo $^{20}$ è chiarissimo: essa contiene non solo del nutrimento cattivo (КaКóv), ma anche del buono e valido ( $\dot{\sigma} \sigma \theta$ $\lambda o ́ v)$. Così l'ascolto-lettura (ảkpóaбıs) dei poeti necessita, perché il giovane possa

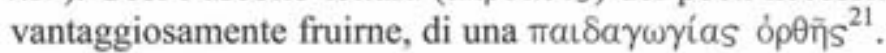

Come si sa, un siffatto tipo di scelta non era nuovo. Si è detto sopra come gli Stoici antichi l'avevano praticata tanto radicalmente da dare il via, o almeno un potente impulso, alla tradizione gnomologica e antologica ${ }^{22}$. Plutarco mette a frutto ampiamente questa metodologia: i poeti devono essere selezionati e la loro opera antologizzata. In particolare, i poeti tragici sono, o meglio possono essere maestri di virtù a patto che si conosca la vera natura della poesia, una natura 'mista', essendo in grado di sceverare il buono dal cattivo, e quindi la si utilizzi, commentandolo come tale.

A tale scopo egli presenta alcuni criteri, che si possono facilmente riassumere in una sorta di formule, quasi dei precetti; ad esempio:

20

21

22

22 Cfr. sopra, alla nota 4. 
Porre sotto diverse rubriche le varie sentenze.

Correggere eventualmente il testo, laddove una affermazione suoni moralmente condannabile, per trasformarlo in una direzione morale ${ }^{23}$. Questo avevano fatto di Stoici e Plutarco raccomanda.

Il poeta stesso spesso fa intendere di disapprovare il contenuto oggettivo di alcuni suoi versi ${ }^{24}$.

Il contesto fornisce talora la chiave appropriata di lettura ${ }^{25}$.

Non possiamo qui addentrarci nell'analisi di questi procedimenti, per altro già ben studiati ${ }^{26}$, e delle loro implicazioni. Vorrei solo accennare un paio di annotazioni conclusive.

Come prima, una osservazione di carattere storico-letterario. Che tipo di libro intende Plutarco raccomandare all'attenzione e alla lettura dei giovani? Li sta forse esortando a leggere le tragedie classiche e si prefigge di insegnare loro a valutarle esteticamente? La risposta, mi sembra, è facilmente negativa.

Plutarco, in questo scritto - conviene ancora una volta sottolinearlo - non si preoccupa di guidare il suo pubblico in una lettura accurata e approfondita di Edipo re o di Medea, o della produzione tragica classica, fornendo ai suoi ascoltatori motivazioni e strumenti in vista della formazione di un giudizio estetico sulla letteratura e sulla tragedia in particolare. Rimangono certamente delusi coloro che si accostano al trattato plutarcheo attendendosi una dettagliata disamina estetica di scritti poetici famosi e accurate costruzioni di poetica teorica. Nello scritto non si trova una parola di valutazione complessiva dei poeti e dei componimenti, un giudizio di valore, una valutazione sulla composizione, nulla. Colpa di una pretesa minorità o ingenuità speculativa dell'estetica antica o, peggio, della manifesta incapacità dell'autore di valutare a fondo la poesia? Risposte di tal genere sono troppo facili e soprattutto troppo intollerabilmente anacronistiche, ed esse sì davvero ingenue, per essere vere.

La soluzione, ritengo, sta da un'altra parte. Si è detto che l'oggetto della riflessione dello scrittore non è un indifferenziato pubblico di persone colte, da indirizzare nell'affinamento del gusto, ma i giovani, e come guidarli nell'apprendere, quasi per gioco, dalla lettura piacevole e scelta dei poeti, quei rudimenti di filosofia, soprattutto di carattere etico, che svilupperanno ed elaboreranno in seguito, attraverso la meditata lettura delle opere teoretiche dei filosofi.

23

24

25

26

Cfr. Plut., De and. poet. cap. 12, 32 E - 34 B.

Cfr. Plut., De aud. poet. cap. 4, 19 A - 22 A. Questo è tanto più vero se si tratta di poesia drammatica o generalmente mimetica.

Cfr. Plut., De aud. poet. cap. 5, 22 A-B.

Un'analisi dettagliata in E. VALGiglıo, Plutarco, De audiendis poetis, Torino, 1973, pp. XXVII-LI. 
A ben guardare, poi, se i giovani e la loro prima educazione sono l'oggetto vero dell'interesse plutarcheo nel De audiendis poetis, non sono nemmeno i giovani in quanto tali i diretti destinatari dello scritto, ma semmai coloro che sono preposti alla loro educazione. Questa educazione comportava l'attenta composizione e il saggio utilizzo di libri di testo per i giovani. Credo che non saremo lontani dal vero se ammettessimo che Plutarco, con il De audiendis poetis, ha inteso comporre una sorta di Einleitung alla lettura di un'antologia appositamente composta per i giovani, una antologia del tipo di quelle che ci testimoniano i papiri almeno fino dall'età ellenistica ${ }^{27}$ e che avranno il loro bacino collettore, secoli dopo, nella raccolta dello Stobeo e in altri testi consimili. In queste antologie il contesto è ovviamente assente e le singole massime dei tragici (e degli altri autori antologizzati) sono estrapolate e riproposte in un ordine escogitato dal compilatore: il più comune è il raggruppamento per argomenti. Questo tratto delle antologie può spiegare, fra l'altro, la raccomandazione che Plutarco rivolge, di contestualizzare cioè i testi ${ }^{28}$.

Fra le altre immediate conseguenze che si possono per noi dedurre dal carattere dell'opera che Plutarco dovette avere in mente è quella, ben nota, dell'obbligo di una grande prudenza ed estrema cautela nell'utilizzazione critico-testuale delle citazioni: si rischia infatti di introdurre nel testo dei tragici lezioni che non appartengono ad esso.

Un'altro punto interessante è quello che si riflette sul dibattuto problema di come il dotto di Cheronea citasse: direttamente da edizioni dei singoli autori o indirettamente?

Se quello che si è delineato è vero, è ovvio che si tratti di un falso problema, almeno nel nostro caso. Se, come credo, Plutarco sta qui commentando un'antologia - reale o forse ideale - è vano chiedersi perché egli sembri talora mostrare di ignorare il contesto di opere che dovrebbero essergli ben familiari. Non è infatti sensato usare, come talora si è fatto, il De audiendis poetis come documento per decidere cosa Plutarco potesse leggere e cosa non più e, per quanto concerne proprio la tragedia, della maggiore o minore conoscenza da parte sua del corpus dei tragici. II tipo di testo che a mio avviso il trattato plutarcheo presuppone non autorizza a questo e in sostanza non può esserci quasi di nessun aiuto in tale ricerca.

Infine una considerazione sulla posizione di Plutarco a proposito del ruolo della poesia e della tragedia in particolare. Come si è detto, dobbiamo stabilire delle distinzioni. La sua posizione di carattere teorico, lo abbiamo visto, è tradizionale:

Si pensi al celebre P.Cair. inv. 65445, il famoso Livre d'écolier du III siècle avant J.-C., edito da O. Guéraud - P. Jouguet, Le Caire 1938, o ai P. Petrie I, 3 (1), P.Hib. I, 7; BKT V, 2, pp. 123 128; BKT V, 2, pp. 129-130, tutti dei primi due secoli dell'età ellenistica.

Cfr. Plut. De and. poet. 5-6, 22 A - 25 B. Spesso una massima "immorale" è infatti pronunciata da un personaggio "immorale" sulla scena. 
nella poesia c'è del buono e del cattivo, e l'antologizzazione consente appunto di sceverare il buono dal cattivo. Esiste tuttavia un altro aspetto, che mi sembra più tipicamente plutarcheo; anch'esso forse non del tutto originale, ma che dovette trovare una profonda consonanza nell'animo e dei convincimenti del Nostro.

Secondo Plutarco, dunque, la poesia è una sorta di pre-filosofia ${ }^{29}$, un riverbero della sapienza, qualche cosa di analogo proprio alla luce lunare, paragonata a quella viva del sole. Ecco dunque perché la poesia è, non solo tollerabile nella pedagogia, ma il suo studio è addirittura auspicabile. Essa, infatti, incarna, per così dire e questo è interessante - lo stadio aurorale della sapienza della nazione, il primo passo verso la filosofia. E' giusto infatti riconoscere che talora i poeti hanno intuito ciò che in seguito i filosofi hanno razionalmente elaborato:

Quando troviamo un qualche elemento in essi (nei poeti) degno e utile, è necessario che lo nutriamo e lo facciamo crescere con le prove e le testimonianze filosofiche, riconoscendone tuttavia ad essi il merito della scoperta ${ }^{30}$.

E' molto utile, dice Plutarco, fare osservare al giovane:

come le dottrine di Pitagora e di Platone si accordino con quanto è detto sulla scena ed è cantato sulla lira ed è studiato nelle scuole e come gli insegnamenti di Chilone e di Biante portino alle stesse idee familiari dalle letture più leggere della giovinezza ${ }^{31}$.

Più avanti poi:

Un tale legame e apparentamento con le dottrine filosofiche strappa la poesia al mito e alla maschera ${ }^{32}$.

Dunque unità sostanziale della cultura nazionale che Plutarco, con notevole acume, sembra percepire, almeno intuire, anche nel suo divenire storico. 
(Página deixada propositadamente em branco) 


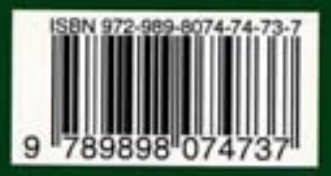

